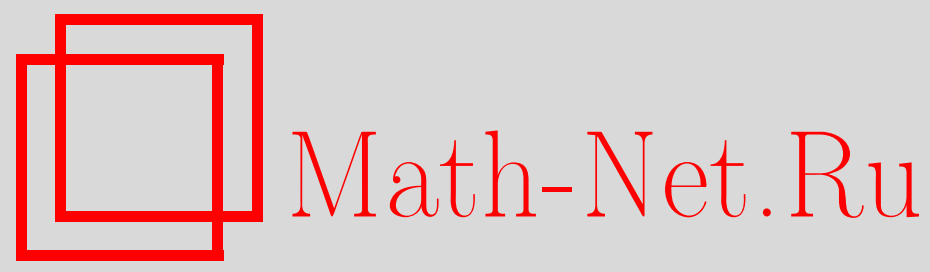

А. М. Зубков, М. П. Савелов, Сходимость последовательности значений статистики Пирсона к квадрату нормированного процесса Бесселя, Дискрет. матем., 2016, том 28, выпуск 3, 49-58

DOI: https://doi.org/10.4213/dm1383

Использование Общероссийского математического портала Math-Net.Ru подразумевает, что вы прочитали и согласны с пользовательским соглашением http://www.mathnet.ru/rus/agreement

Параметры загрузки:

IP : 54.162 .127 .20

26 апреля 2023 г., 13:12:53

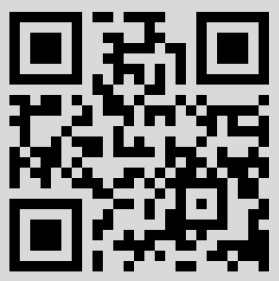




\title{
Сходимость последовательности значений статистики Пирсона к квадрату нормированного процесса Бесселя
}

\author{
() 2016 г. А. М. Зубков*, М. П. Савелов**
}

\begin{abstract}
Показано, что при надлежащей нормировке по времени конечномерные распределения процесса, образованного последовательными значениями статистики Пирсона для выборок увеличивающихся со временем объемов, сходятся к конечномерным распределениям стационарного случайного процесса - нормированного квадрата процесса Бесселя. Полученные ранее результаты о предельных совместных распределениях статистики Пирсона используются для вывода явных формул для плотности совместных распределений процесса Бесселя.
\end{abstract}

Ключевые слова: статистика Пирсона, последовательный критерий хи-квадрат, процесс Бесселя

\section{1. Введение}

Рассмотрим схему независимых испытаний с $N \geqslant 2$ исходами. Будем считать, что вероятность $j$-го исхода равна $p_{j}>0, j=1, \ldots, N$. Для $t=1,2, \ldots$ и $j \in\{1, \ldots, N\}$ введем индикаторы $I_{j}(t): I_{j}(t)=1$, если при $t$-м испытании осуществился $j$-й исход, и $I_{j}(t)=0$ в противном случае. Тогда $\sum_{t=1}^{n} I_{j}(t)-$ частота исхода $j$ в первых $n$ испытаниях. Статистика Пирсона

$$
X(n):=\sum_{j=1}^{N} \frac{1}{n p_{j}}\left(\sum_{t=1}^{n} I_{j}(t)-n p_{j}\right)^{2}
$$

используется, например, в критерии согласия с гипотезой о том, что вероятности наблюдаемых исходов действительно равны $p_{1}, \ldots, p_{N}($ см [9], [5]).

В ряде работ ( [1]- [4]) изучались свойства последовательного критерия хи-квадрат, который строится по статистике $\left(X\left(n_{1}\right), X\left(n_{2}\right), \ldots, X\left(n_{r}\right)\right), n_{1}<n_{2}<$ $\ldots<n_{r}$. В связи с исследованием вероятностей ошибок такого критерия в [1] было доказано, что при любых $0<t_{1}<\ldots<t_{k}<\infty$ и $x_{1}^{*}, \ldots, x_{k}^{*} \geqslant 0$

$$
\lim _{n \rightarrow \infty} \mathbf{P}\left(X\left(\left\lfloor n t_{1}\right\rfloor\right)>x_{1}^{*}, \ldots, X\left(\left\lfloor n t_{k}\right\rfloor\right)>x_{k}^{*}\right)=\int_{\frac{x_{1}^{*}}{2}}^{\infty} \ldots \int_{\frac{x_{k}^{*}}{2}}^{\infty} p_{k}\left(u_{1}, \ldots, u_{k}\right) d u_{1} \ldots d u_{k},
$$

* Место работы: Математический институт им. В. А. Стеклова PAH, e-mail: zubkov@mi.ras.ru

** Место работы: МГУ им. М. В. Ломоносова, e-mail: savelovmp@gmail.com 
где ( [1], формула (4.15) - в наших обозначениях)

$$
\begin{gathered}
p_{k}\left(u_{1}, \ldots, u_{k}\right)= \\
=\left(\frac{t_{k} u_{1} u_{k}}{t_{1}}\right)^{\frac{N-3}{4}} \frac{e^{u_{2}+\ldots+u_{k-1}}}{\Gamma\left(\frac{N-1}{2}\right)} \prod_{i=1}^{k-1} \frac{\exp \left\{-\frac{u_{i}+u_{i+1}}{1-\frac{t_{i}}{t_{i+1}}}\right\}}{1-\frac{t_{i}}{t_{i+1}}} I_{\frac{N-3}{2}}\left(\frac{2 \sqrt{t_{i} t_{i+1} u_{i} u_{i+1}}}{t_{i+1}-t_{i}}\right)
\end{gathered}
$$

и $I_{\delta}(x)$ - функция Инфельда, т. е. модифицированная функция Бесселя первого рода (см., например, [8], [7], с. 139); она является каноническим решением дифференциального уравнения

$$
x^{2} \frac{d^{2} \omega}{d x^{2}}+x \frac{d \omega}{d x}-\left(x^{2}+\delta^{2}\right) \omega=0
$$

разлагается в степенной ряд: $I_{\delta}(x)=\sum_{k=0}^{\infty} \frac{(x / 2)^{2 k+\delta}}{k ! \Gamma(k+\delta+1)}$ и имеет различные интегральные представления.

Рассмотрим случайный процесс с дискретным временем $X(n), n \geqslant 1$.

Теорема 1. Если $1 \leqslant s \leqslant t, m o$

$$
\mathbf{E} X(s)=N-1, \operatorname{cov}(X(s), X(t))=2(N-1) \frac{s}{t}+\frac{1}{t}\left(2-2 N-N^{2}+\sum_{k=1}^{N} \frac{1}{p_{k}}\right) .
$$

В частности, если $s, t \rightarrow \infty$ так, что $\frac{t}{s} \rightarrow C \geqslant 1$, mо

$$
\operatorname{cov}(X(s), X(t))=\frac{2}{C}(N-1)+O\left(t^{-1}\right) .
$$

В силу теоремы 1 дисперсия $\mathbf{D} X(t)=2(N-1)+\frac{1}{t} \cdot\left(2-2 N-N^{2}+\sum_{k=1}^{N} \frac{1}{p_{k}}\right)$; это выражение для дисперсии хорошо известно, см., например, [9], [5], [6].

Следствие 1. Пусть $s \geqslant 1$ фиксировано. Тогда $\operatorname{cov}(X(s), X(t))$ строго монотонно убъвает по $t$ при $t \geqslant s$, за исключением случая $s=1, p_{1}=\ldots=p_{N}=\frac{1}{N}:$ тогда $\operatorname{cov}(X(1), X(t))=0$ при всех $t \geqslant 1$.

Пусть $W_{1}(t), W_{2}(t), \ldots$ - независимые стандартные винеровские процессы, т. е. независимые процессы с независимыми приращениями, $W_{k}(0)=0$ и приращение $W_{k}(t+s)-W_{k}(t)$ имеет нормальное распределение со средним 0 и дисперсией $s$ при любых $k=1,2, \ldots$ и $t, s \geqslant 0$. При любом целом $M>0$ случайный процесс $W^{(M)}(t)=\left(W_{1}(t), \ldots, W_{M}(t)\right)$ является стандартным винеровским $M$-мерным процессом. Случайный процесс

$$
\operatorname{Bes}_{M}(t)=\left\|W^{(M)}(t)\right\|_{2}=\left(\sum_{k=1}^{M} W_{k}^{2}(t)\right)^{1 / 2}
$$

называется $M$-мерным процессом Бесселя; очевидно, $\operatorname{Bes}_{M}^{2}(t)=\sum_{k=1}^{M} W_{k}^{2}(t)$ и поэтому $\frac{1}{t} \operatorname{Bes}_{M}^{2}(t)$ имеет распределение хи-квадрат с $M$ степенями свободы.

Теорема 2. Последовательность случайных процессов $X(\lfloor n t\rfloor), t>0, n p u n \rightarrow \infty$ сходится в смысле конечномерных распределений $\kappa$ процессу $\frac{1}{t} \operatorname{Bes}_{N-1}^{2}(t)$. 
Замечание. Случайный процесс $\widetilde{W}^{(M)}(t)=\left(\widetilde{W}_{1}(t), \ldots, \widetilde{W}_{M}(t)\right) \stackrel{\text { def }}{=} \frac{1}{\sqrt{t}} W^{(M)}(t)$, как и $W^{(M)}(t)$, является гауссовским процессом с нулевым средним, а ковариации между его компонентами равны

$$
\begin{gathered}
\operatorname{Cov}\left(\widetilde{W}_{j}^{(M)}(t), \widetilde{W}_{k}^{(M)}(s)\right)=\operatorname{Cov}\left(\frac{1}{\sqrt{t}} W_{j}(t), \frac{1}{\sqrt{s}}\left(W_{k}(t)+\left(W_{k}(s)-W_{k}(t)\right)\right)\right)= \\
\quad=\operatorname{Cov}\left(\frac{1}{\sqrt{t}} W_{j}(t), \frac{1}{\sqrt{s}} W_{k}(t)\right)=\left\{\begin{array}{ll}
0, & j \neq k, \\
\sqrt{\frac{t}{s}}, & j=k,
\end{array} \quad s>t \geqslant 0 .\right.
\end{gathered}
$$

Случайный процесс $W^{*}(u) \stackrel{\text { def }}{=} \widetilde{W}^{(M)}\left(e^{u}\right), u \in \mathbb{R}$, является стационарным в широком смысле, так как $\mathbf{E} W^{*}(u)=0$ и

$$
\operatorname{Cov}\left(W_{j}^{*}(u), W_{k}^{*}(u+v)\right)=\left\{\begin{array}{ll}
0, & j \neq k, \\
e^{v / 2}, & j=k,
\end{array} \quad v \geqslant 0, u \in \mathbb{R} .\right.
$$

Так как процесс $W^{*}(u)$ гауссовский, то он стационарен и в узком смысле. Следовательно, стационарными в узком смысле являются функции от мгновенных значений процесса $W^{*}(u)$ : процесс $e^{-u} \operatorname{Bes}_{N-1}^{2}\left(e^{u}\right)$ и процесс $e^{-u / 2} \operatorname{Bes}_{N-1}\left(e^{u}\right)$.

Это замечание позволяет переформулировать теорему 2.

Теорема 2а. Конечномерные распределения случайных процессов $X\left(\left\lfloor n e^{t}\right\rfloor\right), t \in \mathbb{R}$, при $n \rightarrow \infty$ сходятся $\kappa$ конечномерным распределениям стационарного в узком смицсле проиесса $e^{-t} \operatorname{Bes}_{N-1}^{2}\left(e^{t}\right)$.

Теорема 2 и формула (1) позволяют легко получить формулы для плотностей конечномерных распределений процесса Бесселя $\operatorname{Bes}_{N}(t)$

Теорема 3. При любом $k \geqslant 2$ и любых $0<t_{1}<\ldots<t_{k}$ совместное распределение $\left(\operatorname{Bes}_{N}\left(t_{1}\right), \ldots, \operatorname{Bes}_{N}\left(t_{k}\right)\right)$ имеет плотность распределения

$$
\begin{gathered}
b_{t_{1}, \ldots, t_{k}}\left(x_{1}, \ldots, x_{k}\right)= \\
=\frac{x_{1}}{t_{1}}\left(\frac{x_{1}^{2} x_{k}^{2}}{4 t_{1}^{2}}\right)^{\frac{N-2}{4}} \frac{e^{\frac{x_{2}^{2}}{2 t_{2}}+\ldots+\frac{x_{k-1}^{2}}{2 t_{k-1}}}}{\Gamma\left(\frac{N}{2}\right)} \prod_{i=1}^{k-1} \frac{x_{i+1}}{t_{i+1}-t_{i}} \exp \left\{-\frac{\frac{x_{i}^{2}}{2 t_{i}}+\frac{x_{i+1}^{2}}{2 t_{i+1}}}{1-\frac{t_{i}}{t_{i+1}}}\right\} I_{\frac{N}{2}-1}\left(\frac{x_{i} x_{i+1}}{t_{i+1}-t_{i}}\right),
\end{gathered}
$$

где $x_{1}, \ldots, x_{k} \geqslant 0 ; b_{t_{1}, \ldots, t_{k}}\left(x_{1}, \ldots, x_{k}\right)=0$, если $\min \left\{x_{1}, \ldots, x_{k}\right\}<0$.

\section{2. Доказательство теоремы 1}

Пусть $I_{j}^{*}(u):=I_{j}(u)-p_{j}$, тогда $X(s)=\sum_{j=1}^{N} \frac{1}{s p_{j}}\left(\sum_{u=1}^{s} I_{j}^{*}(u)\right)^{2}$ и при любых $i, j \in$ $\{1, \ldots, N\}, i \neq j, u \geqslant 1$

$$
\begin{gathered}
\mathbf{E} I_{i}^{*}(u)=0, \quad \mathbf{E}\left(I_{i}^{*}(u)\right)^{2}=p_{i}\left(1-p_{i}\right)^{2}+\left(1-p_{i}\right) p_{i}^{2}=p_{i}\left(1-p_{i}\right), \\
\mathbf{E} I_{i}^{*}(u) I_{j}^{*}(u)=p_{i}\left(1-p_{i}\right)\left(-p_{j}\right)+p_{j}\left(-p_{i}\right)\left(1-p_{j}\right)+\left(1-p_{i}-p_{j}\right) p_{i} p_{j}=-p_{i} p_{j}, \\
\mathbf{E}\left(I_{i}^{*}(u)\right)^{4}=p_{i}\left(1-p_{i}\right)^{4}+\left(1-p_{i}\right) p_{i}^{4}=p_{i}\left(1-p_{i}\right)\left(1-3 p_{i}\left(1-p_{i}\right)\right), \\
\mathbf{E}\left(I_{i}^{*}(u)\right)^{2}\left(I_{j}^{*}(u)\right)^{2}=p_{i}\left(1-p_{i}\right)^{2} p_{j}^{2}+p_{j} p_{i}^{2}\left(1-p_{j}\right)^{2}+\left(1-p_{i}-p_{j}\right) p_{i}^{2} p_{j}^{2}= \\
=p_{i} p_{j}\left(p_{i}+p_{j}-3 p_{i} p_{j}\right) .
\end{gathered}
$$


Из равенств (2) и независимости $I_{i}^{*}(1), I_{i}^{*}(2), \ldots$ следует, что

$$
\begin{aligned}
\mathbf{E} X(s)=\sum_{j=1}^{N} \frac{1}{s p_{j}} \mathbf{E}\left(\sum_{u=1}^{s} I_{j}^{*}(u)\right)^{2}=\mathbf{E} \sum_{j=1}^{N} \frac{1}{s p_{j}} \sum_{u=1}^{s}\left(I_{j}^{*}(u)\right)^{2}= & \\
& =\sum_{j=1}^{N}\left(1-p_{j}\right)=N-1 .
\end{aligned}
$$

Несложно видеть, что (при $1 \leqslant s \leqslant t)$

$$
\begin{gathered}
\mathbf{E} X(s) X(t)=\sum_{i, j=1}^{N} \frac{1}{s p_{i} t p_{j}} \mathbf{E}\left(\sum_{u=1}^{s} I_{i}^{*}(u)\right)^{2}\left(\sum_{v=1}^{t} I_{j}^{*}(v)\right)^{2}= \\
=\sum_{i, j=1}^{N} \frac{1}{s p_{i} t p_{j}} \mathbf{E}\left[\left(\sum_{u=1}^{s}\left(I_{i}^{*}(u)\right)^{2}+2 \sum_{1 \leqslant u_{1}<u_{2} \leqslant s} I_{i}^{*}\left(u_{1}\right) I_{i}^{*}\left(u_{2}\right)\right) \times\right. \\
\left.\times\left(\sum_{v=1}^{t}\left(I_{j}^{*}(v)\right)^{2}+2 \sum_{1 \leqslant v_{1}<v_{2} \leqslant t} I_{j}^{*}\left(v_{1}\right) I_{j}^{*}\left(v_{2}\right)\right)\right]= \\
=\sum_{i, j=1}^{N} \frac{1}{s p_{i} t p_{j}} \sum_{\substack{1 \leqslant u \leqslant s \\
1 \leqslant v \leqslant t}} \mathbf{E}\left(I_{i}^{*}(u)\right)^{2}\left(I_{j}^{*}(v)\right)^{2}+ \\
+4 \sum_{i, j=1}^{N} \frac{1}{s p_{i} t p_{j}} \sum_{\substack{1 \leqslant u_{1}<u_{2} \leqslant s \\
1 \leqslant v_{1}<v_{2} \leqslant t}} \mathbf{E} I_{i}^{*}\left(u_{1}\right) I_{i}^{*}\left(u_{2}\right) I_{j}^{*}\left(v_{1}\right) I_{j}^{*}\left(v_{2}\right),
\end{gathered}
$$

так как остальные слагаемые равны 0 : если $v_{1}<v_{2}$, то $\mathbf{E}\left(I_{i}^{*}(u)\right)^{2} I_{j}^{*}\left(v_{1}\right) I_{j}^{*}\left(v_{2}\right)=0$, поскольку или $v_{1} \neq u$, или $v_{2} \neq u$, и в любом случае один из сомножителей с нулевым математическим ожиданием не зависит от остальных; аналогично $\mathbf{E}\left(I_{j}^{*}(v)\right)^{2} I_{i}^{*}\left(u_{1}\right) I_{i}^{*}\left(u_{2}\right)=0$ при $u_{1}<u_{2}$.

Разобьем первую сумму в правой части (4) на три слагаемых:

$$
\begin{aligned}
& \sum_{i, j=1}^{N} \frac{1}{s p_{i} t p_{j}} \sum_{\substack{1 \leqslant u \leqslant s \\
1 \leqslant v \leqslant t}} \mathbf{E}\left(I_{i}^{*}(u)\right)^{2}\left(I_{j}^{*}(v)\right)^{2}=\sum_{\substack{1 \leqslant i, j \leqslant N \\
i \neq j}} \frac{1}{s p_{i} t p_{j}} \sum_{u=1}^{s} \mathbf{E}\left(I_{i}^{*}(u)\right)^{2}\left(I_{j}^{*}(u)\right)^{2}+ \\
& \quad+\sum_{i=1}^{N} \frac{1}{s p_{i} t p_{i}} \sum_{u=1}^{s} \mathbf{E}\left(I_{i}^{*}(u)\right)^{4}+\sum_{i, j=1}^{N} \frac{1}{s p_{i} t p_{i}} \sum_{\substack{1 \leqslant u \leqslant s \\
1 \leqslant v \leqslant t \\
v \neq u}} \mathbf{E}\left(I_{i}^{*}(u)\right)^{2}\left(I_{j}^{*}(v)\right)^{2} .
\end{aligned}
$$


Первое и второе слагаемые в (5) вычисляются с помощью формул (2):

$$
\begin{gathered}
\sum_{\substack{1 \leqslant i, j \leqslant N \\
i \neq j}} \frac{1}{s p_{i} t p_{j}} \sum_{u=1}^{s} \mathbf{E}\left(I_{i}^{*}(u)\right)^{2}\left(I_{j}^{*}(u)\right)^{2}=\sum_{\substack{1 \leqslant i, j \leqslant N \\
i \neq j}} \frac{s p_{i} p_{j}\left(p_{i}+p_{j}-3 p_{i} p_{j}\right)}{s p_{i} t p_{j}}= \\
=\frac{1}{t} \sum_{\substack{1 \leqslant i, j \leqslant N \\
i \neq j}}\left(p_{i}+p_{j}-3 p_{i} p_{j}\right)=\frac{2(N-1)}{t}-\frac{3}{t}\left(1-\sum_{i=1}^{N} p_{i}^{2}\right), \\
\quad \sum_{i=1}^{N} \frac{1}{s p_{i} t p_{i}} \sum_{u=1}^{s} \mathbf{E}\left(I_{i}^{*}(u)\right)^{4}=\sum_{i=1}^{N} \frac{s p_{i}\left(1-p_{i}\right)\left(1-3 p_{i}\left(1-p_{i}\right)\right)}{s p_{i} t p_{i}}= \\
=\frac{1}{t} \sum_{i=1}^{N} \frac{1-4 p_{i}+6 p_{i}^{2}-3 p_{i}^{3}}{p_{i}}=\frac{1}{t} \sum_{i=1}^{N} \frac{1}{p_{i}}-\frac{4 N}{t}+\frac{6}{t}-\frac{3}{t} \sum_{i=1}^{N} p_{i}^{2} .
\end{gathered}
$$

При вычислении третьего слагаемого в (5) используем еще независимость случайных величин $I_{i}^{*}(u)$ и $I_{j}^{*}(v)$ при $u \neq v$ :

$$
\begin{gathered}
\sum_{i, j=1}^{N} \frac{1}{s p_{i} t p_{i}} \sum_{\substack{1 \leqslant u \leqslant s \\
1 \leqslant v \leqslant t \\
v \neq u}} \mathbf{E}\left(I_{i}^{*}(u)\right)^{2}\left(I_{j}^{*}(v)\right)^{2}=\sum_{i, j=1}^{N} \frac{s(t-1) p_{i}\left(1-p_{i}\right) p_{j}\left(1-p_{j}\right)}{s p_{i} t p_{j}}= \\
=\frac{t-1}{t} \sum_{i, j=1}^{N}\left(1-p_{i}\right)\left(1-p_{j}\right)=\left(1-\frac{1}{t}\right)(N-1)^{2} .
\end{gathered}
$$

Таким образом,

$$
\sum_{i, j=1}^{N} \frac{1}{s p_{i} t p_{j}} \sum_{\substack{1 \leqslant u \leqslant s \\ 1 \leqslant v \leqslant t}} \mathbf{E}\left(I_{i}^{*}(u)\right)^{2}\left(I_{j}^{*}(v)\right)^{2}=(N-1)^{2}+\frac{1}{t}\left[\sum_{i=1}^{N} \frac{1}{p_{i}}-N^{2}\right] .
$$

Рассмотрим вторую сумму в правой части (4). Заметим, что если $1 \leqslant$ $u_{1}<u_{2} \leqslant s, 1 \leqslant v_{1}<v_{2} \leqslant t$ и $\left(u_{1}, u_{2}\right) \neq\left(v_{1}, v_{2}\right)$, то в произведении $I_{i}^{*}\left(u_{1}\right) I_{i}^{*}\left(u_{2}\right) I_{j}^{*}\left(v_{1}\right) I_{j}^{*}\left(v_{2}\right)$ хотя бы один сомножитель не зависит от остальных и поэтому $\mathbf{E} I_{i}^{*}\left(u_{1}\right) I_{i}^{*}\left(u_{2}\right) I_{j}^{*}\left(v_{1}\right) I_{j}^{*}\left(v_{2}\right)=0$. Используя это соображение и формулы $(2)$, находим:

$$
\begin{aligned}
& \sum_{i, j=1}^{N} \frac{1}{s p_{i} t p_{j}} \sum_{\substack{1 \leqslant u_{1}<u_{2} \leqslant s \\
1 \leqslant v_{1}<v_{2} \leqslant t}} \mathbf{E} I_{i}^{*}\left(u_{1}\right) I_{i}^{*}\left(u_{2}\right) I_{j}^{*}\left(v_{1}\right) I_{j}^{*}\left(v_{2}\right)= \\
= & \sum_{i, j=1}^{N} \frac{1}{s p_{i} t p_{j}} \sum_{\substack{1 \leqslant u_{1}<u_{2} \leqslant s\\
}} \mathbf{E} I_{i}^{*}\left(u_{1}\right) I_{j}^{*}\left(u_{1}\right) I_{i}^{*}\left(u_{2}\right) I_{j}^{*}\left(u_{2}\right)= \\
= & \sum_{\substack{1 \leqslant i, j \leqslant N \\
i \neq j}} \frac{1}{s p_{i} t p_{j}} C_{s}^{2} p_{i}^{2} p_{j}^{2}+\sum_{i=1}^{N} \frac{1}{s p_{i} t p_{i}} C_{s}^{2} p_{i}^{2}\left(1-p_{i}\right)^{2}= \\
= & \frac{s-1}{2 t}\left(\sum_{i, j=1}^{N} p_{i} p_{j}+\sum_{i=1}^{N}\left(1-2 p_{i}\right)\right)=\frac{s-1}{2 t}(N-1) .
\end{aligned}
$$


Из последнего равенства, (4), (8) и (3) следует, что при $1 \leqslant s \leqslant t$

$$
\begin{gathered}
\operatorname{cov}(X(s), X(t))=\mathbf{E} X(s) X(t)-\mathbf{E} X(s) \mathbf{E} X(t)= \\
=\frac{1}{t}\left[\sum_{i=1}^{N} \frac{1}{p_{i}}-N^{2}\right]+2 \frac{s-1}{t}(N-1),
\end{gathered}
$$

что совпадает с формулой в утверждении теоремы 1 .

\section{3. Доказательство следствия 1}

Согласно теореме 1

$$
\begin{gathered}
\operatorname{cov}(X(s), X(t))=2(N-1) \frac{s}{t}+\frac{1}{t}\left(2(1-N)-N^{2}+\sum_{i=1}^{N} \frac{1}{p_{i}}\right)= \\
=2(N-1) \frac{s-1}{t}+\frac{1}{t}\left(\sum_{i=1}^{N} \frac{1}{p_{i}}-N^{2}\right) .
\end{gathered}
$$

Очевидно, $2(N-1)(s-1) \geqslant 0$. В силу выпуклости функции $f(p)=\frac{1}{p^{2}}$ и неравенства Иенсена

$$
\sum_{i=1}^{N} \frac{1}{p_{i}}=\sum_{i=1}^{N} p_{i} \frac{1}{p_{i}^{2}} \geqslant\left(\sum_{i=1}^{N} p_{i} \frac{1}{p_{i}}\right)^{2}=N^{2},
$$

Таким образом, оба слагаемых, составляющих $\operatorname{cov}(X(s), X(t))$, неотрицательны и не возрастают по $t$. Их сумма равна 0 только если $s=1 \leqslant t$ и $p_{1}=\ldots=p_{N}=\frac{1}{N}$, в остальных случаях $\operatorname{cov}(X(s), X(t))$ положительна и строго монотонно убывает на множестве $t \geqslant s$.

\section{4. Доказательство теоремы 2}

Положим

$$
\vec{\chi}(u)=\left(\frac{I_{1}^{*}(u)}{\sqrt{p_{1}}}, \ldots, \frac{I_{N}^{*}(u)}{\sqrt{p_{N}}}\right), \quad u=1,2, \ldots
$$

Векторы $\vec{\chi}(u), u=1,2, \ldots$, независимы и одинаково распределены. Носителем их распределения является гиперплоскость $\sqrt{p_{1}} x_{1}+\ldots+\sqrt{p_{N}} x_{N}=0$ в $\mathbb{R}^{N}$. Очевидно, $\mathbf{E} \vec{\chi}(1)=\overrightarrow{0} ;$ из формул $(2)$ легко следует, что матрица ковариаций вектора $\vec{\chi}_{1}$ имеет вид

$$
C_{\chi}=\left(\begin{array}{cccc}
1-p_{1} & -\sqrt{p_{1} p_{2}} & \ldots & -\sqrt{p_{1} p_{N}} \\
-\sqrt{p_{1} p_{2}} & 1-p_{2} & \ldots & -\sqrt{p_{2} p_{N}} \\
\vdots & \vdots & \ddots & \vdots \\
-\sqrt{p_{1} p_{N}} & -\sqrt{p_{2} p_{N}} & \ldots & 1-p_{N}
\end{array}\right)
$$

Лемма. Вектор $\vec{\xi}=\left(\xi_{1}, \ldots, \xi_{N}\right)$, имеющий нормальное распределение в $\mathbb{R}^{N}$ с нулевым средним и матрицей ковариаций $C_{\chi}$, можно представить в виде $\gamma_{1} \vec{e}_{1}^{*}+\ldots+\gamma_{N-1} \vec{e}_{N-1}^{*}$, где $\vec{e}_{1}^{*}, \ldots, \vec{e}_{N-1}^{*}-$ ортонормированный базис в гиперплоскости $\sqrt{p_{1}} x_{1}+\ldots+\sqrt{p_{N}} x_{N}=0, a \gamma_{1}, \ldots, \gamma_{N-1}-$ независимые случайные величины, 
имеющие стандартное нормальное распределение. В частности, $|\vec{\xi}|^{2}=\xi_{1}^{2}+\ldots+\xi_{N}^{2}$ имеет распределение хи-квадрат с $N-1$ степенями свободь.

Доказательство. Пусть вектор $\vec{\Xi}=\left(\Xi_{1}, \ldots, \Xi_{N}\right)$ имеет сферически симметричное нормальное распределение в $\mathbb{R}^{N}$ с единичной матрицей ковариаций. Рассмотрим наряду с исходным базисом $\vec{e}_{1}, \ldots, \vec{e}_{N}$ в $\mathbb{R}^{N}$ ортонормированный базис $\vec{e}_{1}^{*}, \ldots, \vec{e}_{N}^{*}$, в котором $\vec{e}_{N}^{*}=\left(\sqrt{p_{1}}, \ldots, \sqrt{p_{N}}\right)$. Вектор $\vec{\xi}$ можно разложить как по исходному, так и по новому базисам:

$$
\vec{\Xi}=\Xi_{1} \vec{e}_{1}+\ldots+\Xi_{N} \vec{e}_{N}=\gamma_{1} \vec{e}_{1}^{*}+\ldots+\gamma_{N} \vec{e}_{N}^{*}
$$

где $\gamma_{k}=\left(\vec{\Xi}, \vec{e}_{k}^{*}\right), k=1, \ldots, N$.

Так как $\vec{\Xi}$ имеет сферически симметричное нормальное распределение с единичной матрицей ковариаций, а базис $\vec{e}_{1}^{*}, \ldots, \vec{e}_{N}^{*}$ ортонормирован, то $\gamma_{1}, \ldots, \gamma_{N}$ независимы и имеют стандартное нормальное распределение, а вектор $\vec{\gamma}=\gamma_{1} \vec{e}_{1}^{*}+\ldots+$ $\gamma_{N-1} \vec{e}_{N-1}^{*}$ имеет нормальное распределение, носителем которого является гиперплоскость $\sqrt{p_{1}} x_{1}+\ldots+\sqrt{p_{N}} x_{N}=0$.

Очевидно, $\mathbf{M} \vec{\gamma}=\overrightarrow{0}$. Найдем ковариационную матрицу $\vec{\gamma}$. В силу (9) $\vec{\Xi}=\vec{\gamma}+\gamma_{N} \vec{e}_{N}^{*}$ и случайные векторы $\vec{\gamma}$ и $\gamma_{N} \vec{e}_{N}^{*}$ независимы. Так как ковариационная матрица суммы независимых случайных векторов с конечными вторыми моментами равна сумме ковариационных матриц этих векторов, то

$$
\operatorname{Cov}(\vec{\Xi})=E=\operatorname{Cov}(\vec{\gamma})+\operatorname{Cov}\left(\gamma_{N} \vec{e}_{N}^{*}\right)
$$

где $E$ - единичная матрица, и

$$
\operatorname{Cov}\left(\gamma_{N} \vec{e}_{N}^{*}\right)=\operatorname{Cov}\left(\gamma_{N} \sqrt{p_{1}}, \ldots, \gamma_{N} \sqrt{p_{N}}\right)=\left\|\sqrt{p_{i} p_{j}}\right\|_{i, j=1}^{N},
$$

T. e.

$$
\operatorname{Cov}(\vec{\gamma})=E-\left\|\sqrt{p_{i} p_{j}}\right\|_{i, j=1}^{N}
$$

Лемма доказана.

Пусть $0=t_{0}<t_{1}<t_{2}<\ldots<t_{k}$. Из независимости векторов $\vec{\chi}(u)=$ $\left(\frac{I_{1}^{*}(u)}{\sqrt{p_{1}}}, \ldots, \frac{I_{N}^{*}(u)}{\sqrt{p_{N}}}\right), u=1,2, \ldots$, из равенств $\mathbf{E} \vec{\chi}(u)=0, \operatorname{Cov}(\vec{\chi}(u))=C_{\chi}$ и центральной предельной теоремы следует, что при $n \rightarrow \infty$ случайные векторы

$$
\vec{\nu}\left(\left[n t_{i-1}\right],\left[n t_{i}\right]\right)=\sum_{u=\left[n t_{i-1}\right]+1}^{\left[n t_{i}\right]} \vec{\chi}(u)=\left(\frac{1}{\sqrt{p_{i}}} \sum_{u=\left[n t_{i-1}\right]+1}^{\left[n t_{i}\right]} I_{j}^{*}(u)\right)_{j=1}^{N}, \quad i=1, \ldots, k
$$

независимы и асимптотически нормальны с параметрами $\left(\overrightarrow{0}, n\left(t_{i}-t_{i-1}\right) C_{\chi}\right), i=$ $1, \ldots, k$, соответственно. Поэтому совместное распределение нарастающих сумм век- 
торов $\frac{1}{\sqrt{n}} \vec{\nu}\left(\left[n t_{i-1}\right],\left[n t_{i}\right]\right)$

$$
\begin{aligned}
& \frac{1}{\sqrt{n}} \vec{\nu}\left(0,\left[n t_{1}\right]\right)=\left(\frac{1}{\sqrt{n p_{j}}} \sum_{u=1}^{\left[n t_{1}\right]} I_{j}^{*}(u)\right)_{j=1}^{N}, \\
& \frac{1}{\sqrt{n}} \vec{\nu}\left(0,\left[n t_{2}\right]\right)=\frac{1}{\sqrt{n}} \vec{\nu}\left(0,\left[n t_{1}\right]\right)+\frac{1}{\sqrt{n}} \vec{\nu}\left(\left[n t_{1}\right],\left[n t_{2}\right]\right)=\left(\frac{1}{\sqrt{n p_{j}}} \sum_{u=1}^{\left[n t_{2}\right]} I_{j}^{*}(u)\right)_{j=1}^{N}, \ldots, \\
& \frac{1}{\sqrt{n}} \vec{\nu}\left(0,\left[n t_{k}\right]\right)=\sum_{i=1}^{k} \frac{1}{\sqrt{n}} \vec{\nu}\left(\left[n t_{i-1}\right],\left[n t_{i}\right]\right)=\left(\frac{1}{\sqrt{n p_{j}}} \sum_{u=1}^{\left[n t_{k}\right]} I_{j}^{*}(u)\right)_{j=1}^{N},
\end{aligned}
$$

является асимптотически нормальным с нулевым математическим ожиданием и блочной ковариационной матрицей

$$
\left\|\begin{array}{ccccc}
t_{1} C_{\chi} & t_{1} C_{\chi} & t_{1} C_{\chi} & \ldots & t_{1} C_{\chi} \\
t_{1} C_{\chi} & t_{2} C_{\chi} & t_{2} C_{\chi} & \ldots & t_{2} C_{\chi} \\
t_{1} C_{\chi} & t_{2} C_{\chi} & t_{3} C_{\chi} & \ldots & t_{3} C_{\chi} \\
\vdots & \vdots & \vdots & \ddots & \vdots \\
t_{1} C_{\chi} & t_{2} C_{\chi} & t_{3} C_{\chi} & \ldots & t_{k} C_{\chi}
\end{array}\right\|=\left\|\min \left\{t_{i}, t_{j}\right\} C_{\chi}\right\|_{i, j=1}^{k} .
$$

Это распределение совпадает с совместным распределением значений в моменты времени $t_{1}, \ldots, t_{k}$ винеровского процесса $W_{\chi}(t), W_{\chi}(0)=\overrightarrow{0}$, со значениями в $\mathbb{R}^{N}$ с независимыми приращениями, имеющими нормальные распределения: $W_{\chi}(t+u)-$ $W_{\chi}(t) \sim \mathcal{N}\left(\overrightarrow{0}, u C_{\chi}\right)$. Из доказанной выше леммы следует, что процесс $W_{\chi}(t)$ принимает значения в гиперплоскости $\sqrt{p_{1}} x_{1}+\ldots+\sqrt{p_{N}} x_{N}=0$ и в ортонормированном базисе $\vec{e}_{1}^{*}, \ldots, \vec{e}_{N}^{*}$ является стандартным $(N-1)$-мерным винеровским процессом.

Совпадение предельных совместных распределений векторов (11) с конечномерными распределениями $W_{\chi}(t)$ означает, что конечномерные распределения процессов $\frac{1}{\sqrt{n}} \vec{\nu}(0,[n t])=\left(\frac{1}{\sqrt{n p_{j}}} \sum_{u=1}^{[n t]} I_{j}^{*}(u)\right)_{j=1}^{N}, t \geqslant 0$, при $n \rightarrow \infty$ сходятся к конечномерным распределениям процесса $W_{\chi}(t)$, сферически симметричного $(N-1)$-мерного винеровского процесса. Следовательно, конечномерные распределения процессов $\frac{1}{n}|\vec{\nu}(0,[n t])|^{2}=\frac{1}{n} \sum_{j=1}^{N}\left(\frac{1}{p_{j}} \sum_{u=1}^{[n t]} I_{j}^{*}(u)\right)^{2}, t \geqslant 0$ при $n \rightarrow \infty$ сходятся к конечномерным распределениям процесса $\left|W_{\chi}(t)\right|^{2}$, т. е. к распределениям процесса $\operatorname{Bes}_{N-1}^{2}(t)$, являющегося квадратом $(N-1)$-мерного процесса Бесселя $\operatorname{Bes}_{N-1}(t)$.

Наконец, каждый процесс $X([n t])=\sum_{j=1}^{N} \frac{1}{[n t] p_{j}}\left(\sum_{u=1}^{[n t]} I_{j}^{*}(u)\right)^{2}, t \geqslant 0$, отличается от соответствующего процесса $\frac{1}{n}|\vec{\nu}(0,[n t])|^{2}, t \geqslant 0$, только тем, что вместо нормирующего множителя $\frac{1}{n}$ используются зависящие от времени нормирующие множители $\frac{1}{[n t]}$. Поэтому из сходимости при $n \rightarrow \infty$ конечномерных распределений процессов $\frac{1}{n}|\vec{\nu}(0,[n t])|^{2}, t \geqslant 0$, к конечномерным распределениям процесса $\operatorname{Bes}_{N-1}^{2}(t)$ следует сходимость конечномерных распределений процессов $X([n t]), t \geqslant 0$, к конечномерным распределениям процесса $\frac{1}{t} \operatorname{Bes}_{N-1}^{2}(t)$. 


\section{5. Доказательство теоремы 3}

Из формулы (1) и теоремы 2 следует, что при любых $0<t_{1}<\ldots<t_{k}$ и $x_{1}^{*}, \ldots, x_{k}^{*} \geqslant 0$

$$
\begin{gathered}
\mathbf{P}\left(\frac{1}{t_{1}} \operatorname{Bes}_{N-1}^{2}\left(t_{1}\right)>x_{1}^{*}, \ldots, \frac{1}{t_{k}} \operatorname{Bes}_{N-1}^{2}\left(t_{k}\right)>x_{k}^{*}\right)= \\
=\int_{\frac{x_{1}^{*}}{2}}^{\infty} \ldots \int_{\frac{x_{k}^{*}}{2}}^{\infty} p_{k}\left(u_{1}, \ldots, u_{k}\right) d u_{1} \ldots d u_{k} .
\end{gathered}
$$

Так как $\left\{\operatorname{Bes}_{N-1}(t)>x\right\}=\left\{\frac{1}{t} \operatorname{Bes}_{N-1}^{2}(t)>\frac{x^{2}}{t}\right\}$ при $x \geqslant 0$, то

$$
\mathbf{P}\left(\operatorname{Bes}_{N-1}\left(t_{1}\right)>x_{1}, \ldots, \operatorname{Bes}_{N-1}\left(t_{k}\right)>x_{k}\right)=\int_{\frac{x_{1}^{2}}{2 t_{1}}}^{\infty} \ldots \int_{\frac{x_{k}^{2}}{2 t_{k}}}^{\infty} p_{k}\left(u_{1}, \ldots, u_{k}\right) d u_{1} \ldots d u_{k}
$$

Поэтому плотность $b_{t_{1}, \ldots, t_{k}}\left(x_{1}, \ldots, x_{k}\right)$ совместного распределения $\left(\operatorname{Bes}_{N-1}\left(t_{1}\right), \ldots, \operatorname{Bes}_{N-1}\left(t_{k}\right)\right)$ имеет вид

$$
\begin{aligned}
& b_{t_{1}, \ldots, t_{k}}\left(x_{1}, \ldots, x_{k}\right)=p_{k}\left(\frac{x_{1}^{2}}{2 t_{1}}, \ldots, \frac{x_{k}^{2}}{2 t_{k}}\right) \frac{x_{1} \ldots x_{k}}{t_{1} \ldots t_{k}}= \\
& =\frac{x_{1} \ldots x_{k}}{t_{1} \ldots t_{k}}\left(\frac{x_{1}^{2} x_{k}^{2}}{4 t_{1}^{2}}\right)^{\frac{N-3}{4}} \frac{e^{\frac{x_{2}^{2}}{2 t_{2}}+\ldots+\frac{x_{k-1}^{2}}{2 t_{k-1}}}}{\Gamma\left(\frac{N-1}{2}\right)} \prod_{i=1}^{k-1} \frac{\exp \left\{-\frac{\frac{x_{i}^{2}}{2 t_{i}}+\frac{x_{i+1}^{2}}{2 t_{i+1}}}{1-\frac{t_{i}}{t_{i+1}}}\right\}}{\left(1-\frac{t_{i}}{t_{i+1}}\right)} I_{\frac{N-3}{2}}\left(\frac{\sqrt{t_{i} t_{i+1} \frac{x_{i}^{2} x_{i+1}^{2}}{t_{i} t_{i+1}}}}{t_{i+1}-t_{i}}\right)= \\
& =\frac{x_{1}}{t_{1}}\left(\frac{x_{1}^{2} x_{k}^{2}}{4 t_{1}^{2}}\right)^{\frac{N-3}{4}} \frac{e^{\frac{x_{2}^{2}}{2 t_{2}}+\ldots+\frac{x_{k-1}^{2}}{2 t_{k-1}}}}{\Gamma\left(\frac{N-1}{2}\right)} \prod_{i=1}^{k-1} \frac{x_{i+1}}{t_{i+1}-t_{i}} \exp \left\{-\frac{\frac{x_{i}^{2}}{2 t_{i}}+\frac{x_{i+1}^{2}}{2 t_{i+1}}}{1-\frac{t_{i}}{t_{i+1}}}\right\} I_{\frac{N-3}{2}}\left(\frac{x_{i} x_{i+1}}{t_{i+1}-t_{i}}\right) \text {. }
\end{aligned}
$$

Для завершения доказательства теоремы 3 остается заменить в этой формуле $N$ на $N+1$.

\section{Список литературы}

1. Захаров В.К., Сарманов О.В., Севастьянов Б.А., "Последовательный критерий $\chi^{2}$ ", Матем. сб., 79(121):3(7) (1969), 444-460; англ. пер.: Zakharov V. K., Sarmanov O. V., Sevast'yanov B. A., "Sequential $\chi^{2}$ criteria", Math. USSR-Sb., 8:3 (1969), 419-435.

2. Селиванов Б.И., Чистяков В.П., "Многомерное распределение хи-квадрат для неоднородной полиномиальной схемы", Дискрет. матем., 10:2 (1998), 52-61; англ. пер.: Selivanov B. I., Chistyakov V. P., "Multivariate chi-square distribution for non-homogeneous polynomial scheme", Discrete Math. Appl., 8:3 (1998), 263-273.

3. Ронжин А.Ф., "Предельное распределение процесса хи-квадрат при наличии разладки", Теория вероятн. и ее примен., 29:3 (1984), 590-594; англ. пер.: Ronzhin A. F., "Тhe limit distribution for a chi-square process with disorder", Theory Probab. Appl., 29:3 (1985), 613-617.

4. Гермогенов А.П., Ронжин А.Ф., "Последовательный критерий хи-квадрат", Теория вероятн. и ее примен., 29:2 (1984), 387-392; англ. пер.: Germogenov A. P., Ronzhin A. F., "A sequential chi-square test", Theory Probab. Appl., 29:2 (1985), 397-403. 
5. Туманян С.Х., “Асимптотическое распределение критерия $\chi^{2}$ при одновременном возрастании объема наблюдений и числа групп”, Теория вероятн. и ее примен., 1:1 (1956), 131-145; англ. пер.: Tumanyan S. Kh., "Asymptotic distribution of the $\chi^{2}$ criterion when the number of observations and number of groups increase simultaneously", Theory Probab. Appl., 1:1 (1956), 117-131.

6. Боровков А.А., Математическая статистика, СПб.: Лань, 2010, 704 с.; англ. пер.: Borovkov A. A., Mathematical statistics, Gordon and Breach, 1998, 592 pp.

7. Лебедев Н.Н., Специальные функиии и их приложения, М.: ФИЗМАТЛИТ, 1963, 359 с.; англ. пер.: Lebedev N. N., Special Functions \& Their Applications, Courier Corporation, 2012, 308 pp.

8. Ватсон Г.Н., Теория бесселевых функиий, М.: Изд. иностр. лит., 1949, 800 с.; пер. с англ.: Watson G. N., A treatise on the theory of Bessel functions, Cambridge University Press, Cambridge, 1922.

9. Крамер Г., Математические методы статистики, М.: Мир, 1975, 648 с.; пер. с англ.: Cramer H., Mathematical Methods of Statistics, Princeton Math. Ser., Uppsala: Almqvist \& Wiksells, 1945, 575 pp.

Статья поступила 24.06.2016. 